\title{
Models and Algorithms for Constructing a Formalized Description of Production Technologies
}

\author{
Dmitry Andreev \\ Faculty of Computer Science and \\ Electrical Power Engineering \\ Pskov State University \\ Pskov, Russia \\ dandreev60@mail.ru \\ Sergey Verteshev \\ Faculty of Computer Science and \\ Electrical Power Engineering \\ Pskov State University \\ Pskov, Russia \\ verteshev@mail.ru
}

\author{
Sergey Lyokhin \\ Faculty of Computer Science and \\ Electrical Power Engineering \\ Pskov State University \\ Pskov, Russia \\ slyokhin@gmail.com
}

\author{
Lilia Motaylenko \\ Faculty of Computer Science and \\ Electrical Power Engineering \\ Pskov State University \\ Pskov, Russia \\ lvs_@mail.ru
}

\begin{abstract}
The problem of technological knowledge formalization is being considered. Discussed features of systems in the scope of ontological engineering of processes, applied to solving the problems of the structural representation of technologies associated with the organization of their analysis. Proposed a model of the concept of technological action, which identifies technological actions as holistic conceptual formations. Constructed the model of formalized description of technologies, which provides unambiguous transition from verbal descriptions of technologies to their ontological representations. Described algorithms for constructing a formalized description of technologies, which implement the logical procedures for automated and automatic construction of ontological representations of technologies. Concluded in the development of a new method of formalized description of technologies, the fundamental principles of which constitute the distinctive features of the proposed models and constructed algorithms.
\end{abstract}

Keywords-algorithmic procedure, relation, technological action.

\section{INTRODUCTION}

In the modern world technological knowledge with full confidence can be considered as the most valuable information. This is because of the increased intensity of a struggle for possession of information resources, among which the knowledge of production technologies (hereinafter - technologies) occupies one of the leading positions [1] - [4]. The presence of a huge number of technologies that differ in the most diverse groups of their components makes it necessary to analyze and compare them. Solution of scientific and practical problems within the framework of this problem receives a new impulse of development in the presence of effective tools for constructing a formalized description of technologies.

At present, the fixation of knowledge about technologies occurs through various methods of their description, which are quite heterogeneous from the standpoint of formalization aspects [5]. At the same time, the possibility of computer processing of this knowledge in the interests of analysis of technologies is significantly limited by the means of implementing these methods. In this regard, a trend towards a more efficient use of technological knowledge is needed to overcome this situation, which can be ensured by their successful operation within a progressive methodological basis, the role of which in recent decades has been actively claimed by the concept of a system-ontological approach [6] - [9].

The study of the features of systems in the field of ontological engineering of processes shows the impossibility of their application to solving the problems of formalized representation of technological knowledge associated with the organization of analysis of technologies. Problem arises in weak elaboration of formal mechanisms of the applied level of description of technologies, in the part of the formation of their decomposition structures (DST) that is expressed in the absence of representation of the activity element as a holistic conceptual formation and in the replacement of the axiomatics of ontological models with graphical notations of the corresponding tools [10], [11].

Thus, it seems to be very important to develop a method of formalized description of technologies by means of a system-ontological approach, the basic principles of which allows operating technological knowledge by computer tools in automated and automatic modes and solving a whole range of tasks of analysis of technologies. 
II. THE MODEL OF THE CONCEPT OF TECHNOLOGICAL ACTION

From the semantic point of view, the ontology should store information about the sets of concepts, whose purpose is to conduct a detailed formalization of certain field of knowledge [12]. In accordance with the generally accepted practice of ontological modeling, conceptual elements of the field of knowledge are required to act as such concepts. The features of this research indicate the need to consider such concept as the concept of technological action, which is a single holistic formation of a formalized description of technology [13].

The model of the concept of technological action is the basic representation of each of the technological actions located in the nodes of the DST, is proposed to be represented by a tuple [14]:

$$
\dot{T D_{u}}=<\dot{T \dot{P}_{u}}, Y_{u}, \dot{X}_{u} \mid \dot{W}_{u}, H_{u}, Z_{u}>
$$

where $T D_{u}$ - a concept of technological action, $T P_{u}$ - a kernel of the concept, $Y_{u}$ - a set of resulting components, $X_{u}$ - a set of source components, $W_{u}$ - a set of invariant components, $H_{u}$ - a set of cost characteristics, $Z_{u}-$ a set of own characteristics.

When designing the DST, the following initial degrees of content formation of the concepts are determined:

Definition 1. The concept is fully formed if

$$
\begin{aligned}
& \forall T D_{u}\left(T P_{u} \neq \varnothing, Y_{u} \neq \varnothing, X_{u} \neq \varnothing,\right. \\
& \left.W_{u} \neq \varnothing, H_{u} \neq \varnothing, Z_{u} \neq \varnothing \vee Z_{u}=\varnothing\right)
\end{aligned}
$$

Definition 2. The concept is preliminary formed if

$$
\begin{aligned}
& \forall T D_{u}^{\prime}\left(T P_{u}^{\prime} \neq \varnothing, Y_{u}^{\prime} \neq \varnothing, X_{u}^{\prime}=\varnothing,\right. \\
& \left.W_{u}^{\prime}=\varnothing, H_{u}^{\prime}=\varnothing, Z_{u}^{\prime}=\varnothing\right)
\end{aligned}
$$

The designation $u$ represents the quantity of positions in the index of each of the technological actions located in the nodes of the DST. The index is an ordered, well-defined sequence of natural numbers, the order of formation of which is that the set of values of each subsequent position is put in accordance with a specific element of the set of values of the previous position [15]. Thus, the unambiguous identification of the location of the concepts of technological actions in the nodes of the DST is formalized. For example, for a certain concept of $\lambda$-level of the DST, the index $u$ is the sequence of natural numbers $\left(\alpha_{i}, \beta_{j}, \ldots, \eta_{l}, \lambda_{m}\right)$. If this concept is preliminary formed, then it can be detailed to the presentation in the form of a set of other concepts, the index of each of which will be supplemented by an additional position. Such formation is also called a "unified decomposition construction" (UDC), at the apex of which is the only holistic concept, and at the basis is a set of private concepts. "Fig. 1" shows an example of one of the possible variants of the graphical view of the UDC.

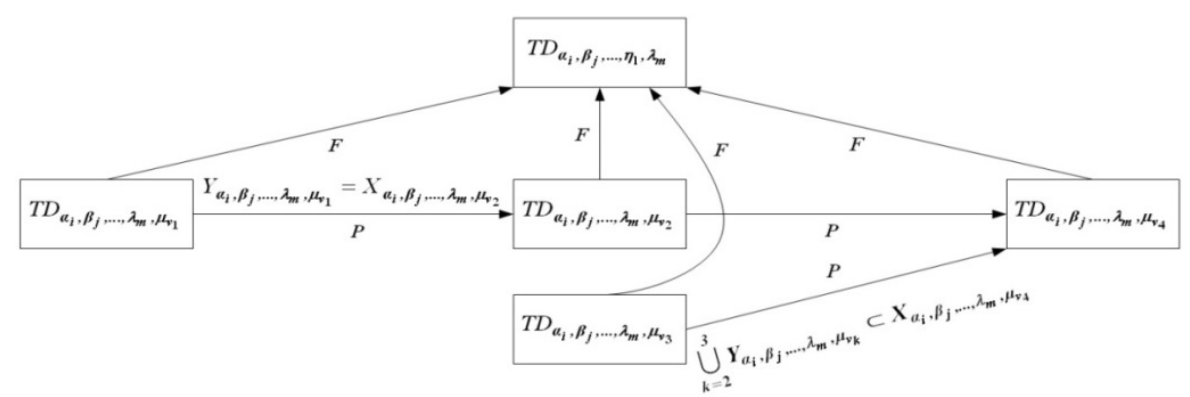

Fig. 1. The example of the UDC

III. THE MODEL OF THE FORMALIZED DESCRIPTION OF TECHNOLOGIES

In information technologies and computer science, ontology is most often considered as an explicit specification of conceptualization, which implies the formalization of a certain field of knowledge not only through the presence of a set of concepts, but also the description of relations between them [16].

In order to describe relations between the fully formed private concepts of the same level of decomposition, the relation $P$ of "immediate precedence" is introduced [17].

Definition 3. Fully formed concepts are in the relation of immediate precedence if

$$
\begin{aligned}
& \forall T D_{\alpha_{i}, \beta_{j}, \ldots, \lambda_{m}, \mu_{r}} \forall T D_{\alpha_{i}, \beta_{j}, \ldots, \lambda_{m}, \mu_{s}} \\
& \left(T D_{\alpha_{i}, \beta_{j}, \ldots, \lambda_{m}, \mu_{r}} P T D_{\alpha_{i}, \beta_{j}, \ldots, \lambda_{m}, \mu_{s}}\right): \\
& :\left(Y_{\alpha_{i}, \beta_{j}, \ldots, \lambda_{m}, \mu_{r}} \subseteq X_{\alpha_{i}, \beta_{j}, \ldots, \lambda_{m}, \mu_{s}}\right)
\end{aligned}
$$

When designing a DST, each of the levels of decomposition will have so-called "initial" and "finite", and some of them will also have "complementary" fully formed concepts.

Definition 4. A fully formed concept of a certain level of decomposition is initial if

$$
\begin{aligned}
& \forall T D_{\alpha_{i}, \beta_{j}, \ldots, \lambda_{m}, \mu_{r}} \\
& \exists T D_{\alpha_{i}, \beta_{j}, \ldots, \lambda_{m}, \mu_{s}} \exists T D_{\alpha_{i}, \beta_{j}, \ldots, \lambda_{m}, \mu_{q}} \\
& \left(\left(Y_{\alpha_{i}, \beta_{j}, \ldots, \lambda_{m}, \mu_{r}} \subseteq X_{\alpha_{i}, \beta_{j}, \ldots, \lambda_{m}, \mu_{s}}\right) \wedge\right. \\
& \left.\wedge\left(Y_{\alpha_{i}, \beta_{j}, \ldots, \lambda_{m}, \mu_{q}} \subseteq X_{\alpha_{i}, \beta_{j}, \ldots, \lambda_{m}, \mu_{r}}\right)\right)
\end{aligned}
$$

Definition 5. A fully formed concept of a certain level of decomposition is finite if

$$
\begin{aligned}
& \forall T D_{\alpha_{i}, \beta_{j}, \ldots, \lambda_{m}, \mu_{r}} \\
& \exists T D_{\alpha_{i}, \beta_{j}, \ldots, \lambda_{m}, \mu_{q}} \bar{\exists} T D_{\alpha_{i}, \beta_{j}, \ldots, \lambda_{m}, \mu_{s}} \\
& \left(\left(Y_{\alpha_{i}, \beta_{j}, \ldots, \lambda_{m}, \mu_{q}} \subseteq X_{\alpha_{i}, \beta_{j}, \ldots, \lambda_{m}, \mu_{r}}\right) \wedge\right. \\
& \left.\wedge\left(Y_{\alpha_{i}, \beta_{j}, \ldots, \lambda_{m}, \mu_{r}} \subseteq X_{\alpha_{i}, \beta_{j}, \ldots, \lambda_{m}, \mu_{s}}\right)\right)
\end{aligned}
$$


Definition 6. A fully formed concept is complementary to another fully formed concept related to the same level of decomposition if

$$
\begin{aligned}
& \forall T D_{\alpha_{i}, \beta_{j}, \ldots, \lambda_{m}, \mu_{t}} \exists T D_{\alpha_{i}, \beta_{j}, \ldots, \lambda_{m}, \mu_{s}} \\
& \exists T D_{\alpha_{i}, \beta_{j}, \ldots, \lambda_{m}, \mu_{r}} \exists T D_{\alpha_{i}, \beta_{j}, \ldots, \lambda_{m}, \mu_{q}} \\
& \bar{\exists} T D_{\alpha_{i}, \beta_{j}, \ldots, \lambda_{m}, \mu_{u}}\left(\left(Y_{\alpha_{i}, \beta_{j}, \ldots, \lambda_{m}, \mu_{t}} \subset\right.\right. \\
& \left.\subset X_{\alpha_{i}, \beta_{j}, \ldots, \lambda_{m}, \mu_{s}}\right) \wedge\left(Y_{\alpha_{i}, \beta_{j}, \ldots, \lambda_{m}, \mu_{r}} \subseteq\right. \\
& \left.\subseteq\left(X_{\alpha_{i}, \beta_{j}, \ldots, \lambda_{m}, \mu_{s}} \backslash X_{\alpha_{i}, \beta_{j}, \ldots, \lambda_{m}, \mu_{s}}^{*}\right)\right) \wedge \\
& \wedge\left(Y_{\alpha_{i}, \beta_{j}, \ldots, \lambda_{m}, \mu_{q}} \subseteq X_{\alpha_{i}, \beta_{j}, \ldots, \lambda_{m}, \mu_{r}}\right) \wedge \\
& \left.\wedge\left(Y_{\alpha_{i}, \beta_{j}, \ldots, \lambda_{m}, \mu_{u_{u}}} \subseteq X_{\alpha_{i}, \beta_{j}, \ldots, \lambda_{m}, \mu_{t}}\right)\right)
\end{aligned}
$$

In order to describe relations between each of the fully formed private concepts and the already fully formed holistic concept, the relation $F$ of "part-whole" is introduced [18].

Definition 7 . Fully formed concepts are in the relation of "part-whole" if (8).

The ordered totality of this kind of UDC forms DST.

Then the model of the formalized description of technologies is determined by the tuple [19]:

$$
\text { Ont }_{\text {Tech }}=<T D, P, F>\text {, }
$$

where $T D=\mathrm{Y} T D_{u}-$ the set of concepts of technological actions, $P$ - the intra-level relation of immediate precedence, $F$ - the inter-level relation of "part-whole".

$$
\begin{aligned}
& \forall T D_{\alpha_{i}, \beta_{j}, \ldots, \lambda_{m}, \mu_{r}} \forall T D_{\alpha_{i}, \beta_{j}, \ldots, \eta_{l}, \lambda_{m}} \\
& \left(T D_{\alpha_{i}, \beta_{j}, \ldots, \lambda_{m}, \mu_{r}} F T D_{\alpha_{i}, \beta_{j}, \ldots, \eta_{l}, \lambda_{m}}\right): \\
& :\left(Y_{\alpha_{i}, \beta_{j}, \ldots, \lambda_{m}, \mu_{r}} \subset Y_{\alpha_{i}, \beta_{j}, \ldots, \eta_{l}, \lambda_{m}}\right) \wedge \\
& \wedge\left(\left(\left(W_{\alpha_{i}, \beta_{j}, \ldots, \lambda_{m}, \mu_{r}}=Y_{k=1}^{n} w_{\alpha_{i}, \beta_{j}, \ldots, \lambda_{m}, \mu_{r}}^{k}\right) \subset\right.\right. \\
& \left.\subset\left(W_{\alpha_{i}, \beta_{j}, \ldots, \eta_{l}, \lambda_{m}}=Y_{k=1}^{n} w_{a_{i}, \beta_{j}, \ldots, l_{l}, \lambda_{m}}^{k}\right)\right) \mid \\
& \mid \exists w_{\alpha_{i}, \beta_{j}, \ldots, \lambda_{m}, u_{r}}^{k_{a}} \exists w_{\alpha_{i}, \beta_{j}, \ldots, \eta, \lambda_{m}}^{k_{a}}, \\
& \left.\left(w_{a_{i}, \beta_{j}, \ldots, \lambda_{m}, \mu_{m}}^{k_{a}} \subset w_{a_{i}, \beta_{j}, \ldots, \lambda_{m}}^{k_{a}}\right)\right) \wedge \\
& \wedge\left(\left(X_{\alpha_{i}, \beta_{j}, \ldots, \lambda_{m}, \mu_{r}} \subset X_{\alpha_{i}, \beta_{j}, \ldots, \eta_{l}, \lambda_{m}}\right) \vee\right. \\
& \vee\left(\left(X_{\alpha_{i}, \beta_{j}, \ldots, \lambda_{m}, \mu_{r}} \backslash X_{\alpha_{i}, \beta_{j}, \ldots, \lambda_{m}, \mu_{r}}^{*}\right) \subset\right. \\
& \left.\subset X_{\alpha_{i}, \beta_{j}, \ldots, \eta_{l}, \lambda_{m}}\right) \vee \\
& \left.\vee\left(\neg\left(X_{\alpha_{i}, \beta_{j}, \ldots, \lambda_{m}, \mu_{r}} \subset X_{\alpha_{i}, \beta_{j}, \ldots, \eta_{l}, \lambda_{m}}\right)\right)\right)
\end{aligned}
$$

The proposed model of the form (9) allows making a set-theoretic analysis of the considered descriptions of technologies by means of the following set of axiomatic properties [20]:
1 . The set of resulting components of a fully formed concept is unique:

$$
\begin{aligned}
& \forall T D_{\alpha_{i}, \beta_{j}, \ldots, \lambda_{m}, \mu_{r}} \forall T D_{\alpha_{i}, \beta_{j}, \ldots, \lambda_{m}, \mu_{s}} \\
& \left(Y_{\alpha_{i}, \beta_{j}, \ldots, \lambda_{m}, \mu_{r}} \mathrm{I} Y_{\alpha_{i}, \beta_{j}, \ldots, \lambda_{m}, \mu_{s}}=\varnothing\right)
\end{aligned}
$$

2. The sets of source and resulting components of a fully formed concept do not have common elements:

$$
\begin{aligned}
& \forall T D_{\alpha_{i}, \beta_{j}, \ldots, \lambda_{m}, \mu_{r}} \\
& \left(X_{\alpha_{i}, \beta_{j}, \ldots, \lambda_{m}, \mu_{r}} \mathrm{I} Y_{\alpha_{i}, \beta_{j}, \ldots, \lambda_{m}, \mu_{r}}=\varnothing\right)
\end{aligned}
$$

3. On the sign of the decomposition of the kernel of a preliminary formed holistic concept:

$$
\begin{aligned}
& \exists T D_{\alpha_{i}, \beta_{j}, \ldots, \eta_{l}, \lambda_{m}}^{\prime} \exists \underset{\mu}{\mathrm{Y} T D_{\alpha_{i}, \beta_{j}, \ldots, \lambda_{m}, \mu}^{\prime}} \\
& \left(\left(Y_{\alpha_{i}, \beta_{j}, \ldots, \eta_{l}, \lambda_{m}}^{\prime}=\mathrm{Y}_{\mu} Y_{\alpha_{i}, \beta_{j}, \ldots, \lambda_{m}, \mu}^{\prime} \mid\right.\right. \\
& \mid \mu=\overline{1, n}, n \geq 2) \Rightarrow \\
& \Rightarrow\left(T P_{\alpha_{i}, \beta_{j}, \ldots, \eta_{l}, \lambda_{m}}^{\prime}=\mathrm{Y}_{\mu} T P_{\alpha_{i}, \beta_{j}, \ldots, \lambda_{m}, \mu}^{\prime} \mid\right. \\
& \mid \mu=\overline{1, n}, n \geq 2))
\end{aligned}
$$

4. On the definition of a complement to a set of source components of a fully formed concept:

$$
\begin{aligned}
& \forall T D_{\alpha_{i}, \beta_{j}, \ldots, \lambda_{m}, \mu_{r}} \forall T D_{\alpha_{i}, \beta_{j}, \ldots, \lambda_{m}, \mu_{s}} \\
& \left(\left(Y_{\alpha_{i}, \beta_{j}, \ldots, \lambda_{m}, \mu_{r}} \subset X_{\alpha_{i}, \beta_{j}, \ldots, \lambda_{m}, \mu_{s}}\right) \Rightarrow\right. \\
& \Rightarrow\left(\exists Y_{k=1}^{n} T D_{\alpha_{i}, \beta_{j}, \ldots, \lambda_{m}, \mu_{v_{k}}} \vee\right. \\
& \vee \exists Y_{k=1}^{n} T D_{\alpha_{i}, \beta_{j}, \ldots, \lambda_{m}, \mu_{v_{k}}}, \\
& Y_{k=1}^{n} Y_{\alpha_{i}, \beta_{j}, \ldots, \lambda_{m}, \mu_{v_{k}}} \subseteq \\
& \left.\left.\subseteq\left(X_{\alpha_{i}, \beta_{j}, \ldots, \lambda_{m}, \mu_{s}} \backslash X_{\alpha_{i}, \beta_{i}, \ldots, \lambda_{m}, \mu_{s}}^{*}\right)\right)\right)
\end{aligned}
$$

5. The set of resulting components of a fully formed concept cannot be a subset of several sets of source components of other fully formed concepts:

$$
\begin{aligned}
& \forall T D_{\alpha_{i}, \beta_{j}, \ldots, \lambda_{m}, \mu_{r}} \\
& \exists T D_{\alpha_{i}, \beta_{j}, \ldots, \lambda_{m}, \mu_{s}} \exists T D_{\alpha_{i}, \beta_{j}, \ldots, \lambda_{m}, \mu_{q}} \\
& \left(\left(Y_{\alpha_{i}, \beta_{j}, \ldots, \lambda_{m}, \mu_{r}} \subseteq X_{\alpha_{i}, \beta_{j}, \ldots, \lambda_{m}, \mu_{s}}\right) \wedge\right. \\
& \left.\wedge\left(Y_{\alpha_{i}, \beta_{j}, \ldots, \lambda_{m}, \mu_{r}} \subseteq X_{\alpha_{i}, \beta_{j}, \ldots, \lambda_{m}, \mu_{q}}\right)\right)
\end{aligned}
$$

6. On the definition of a fully formed holistic concept on the basis an aggregated set of source components: 


$$
\begin{aligned}
& \exists T D_{\alpha_{i}, \beta_{j}, \ldots, \eta_{l}, \lambda_{m}}^{\prime} \exists \mathrm{Y}_{\mu} T D_{\alpha_{i}, \beta_{j}, \ldots, \lambda_{m}, \mu} \\
& \left(\left(\mathrm{Y}_{\mu} X_{\alpha_{i}, \beta_{j}, \ldots, \lambda_{m}, \mu}=X_{\alpha_{i}, \beta_{j}, \ldots, \eta_{l}, \lambda_{m}}\right) \mid\right. \\
& \mid\left(\forall T D _ { \alpha _ { i } , \beta _ { j } , \ldots , \lambda _ { m } , \mu _ { \mathrm { r } } } \left(\bar{\exists} T D_{\alpha_{i}, \beta_{j}, \ldots, \lambda_{m}, \mu_{q}}\right.\right. \\
& \left(\left(Y_{\alpha_{i}, \beta_{j}, \ldots, \lambda_{m}, \mu_{q}} \subseteq X_{\alpha_{i}, \beta_{j}, \ldots, \lambda_{m}, \mu_{r}}\right) \Rightarrow\right. \\
& \Rightarrow\left(X_{\alpha_{i}, \beta_{j}, \ldots, \eta_{l}, \lambda_{m}}:=X_{\alpha_{i}, \beta_{j}, \ldots, \eta_{l}, \lambda_{m}} \cup\right. \\
& \left.\left.\cup X_{\alpha_{i}, \beta_{j}, \ldots, \lambda_{m}, \mu_{r}}\right)\right) \vee \exists T D_{\alpha_{i}, \beta_{j}, \ldots, \lambda_{m}, \mu_{q}} \\
& \left(\left(\left(Y_{\alpha_{i}, \beta_{j}, \ldots, \lambda_{m}, \mu_{q}} \subset X_{\alpha_{i}, \beta_{j}, \ldots, \lambda_{m}, \mu_{r}}\right) \Rightarrow\right.\right. \\
& \Rightarrow\left(X_{\alpha_{i}, \beta_{j}, \ldots, \eta_{l}, \lambda_{m}}:=X_{\alpha_{i}, \beta_{j}, \ldots, \eta_{l}, \lambda_{m}} \cup\right. \\
& \left.\left.\cup\left(X_{\alpha_{i}, \beta_{j}, \ldots, \lambda_{m}, \mu_{r}} \backslash X_{\alpha_{i}, \beta_{j}, \ldots, \lambda_{m}, \mu_{r}}^{*}\right)\right)\right) \vee \\
& \vee\left(\left(Y_{\alpha_{i}, \beta_{j}, \ldots, \lambda_{m}, \mu_{q}}=X_{\alpha_{i}, \beta_{j}, \ldots, \lambda_{m}, \mu_{r}}\right) \Rightarrow\right. \\
& \Rightarrow\left(X_{\alpha_{i}, \beta_{j}, \ldots, \eta_{l}, \lambda_{m}}:=X_{\alpha_{i}, \beta_{j}, \ldots, \eta_{l}, \lambda_{m}} \cup\right. \\
& \cup \varnothing)))) \Rightarrow\left(T D_{\alpha_{i}, \beta_{j}, \ldots, \eta_{l}, \lambda_{m}}^{\prime} \rightarrow\right. \\
& \left.\left.\rightarrow T D_{\alpha_{i}, \beta_{j}, \ldots, \eta_{l}, \lambda_{m}}\right)^{\mathrm{X}}\right)
\end{aligned}
$$

7. On the definition of a fully formed holistic concept on the basis an aggregated set of invariant components:

$$
\begin{aligned}
& \exists T D_{\alpha_{i}, \beta_{j}, \ldots, \eta_{l}, \lambda_{m}}^{\prime} \exists \mathrm{Y}_{\mu} T D_{\alpha_{i}, \beta_{j}, \ldots, \lambda_{m}, \mu} \\
& \left(\left(\mathrm{Y}_{\mu} W_{\alpha_{i}, \beta_{j}, \ldots, \lambda_{m}, \mu}=W_{\alpha_{i}, \beta_{j}, \ldots, \eta_{l}, \lambda_{m}}\right) \mid\right. \\
& \mid\left(\forall T D_{\alpha_{i}, \beta_{j}, \ldots, \lambda_{m}, \mu_{\mathrm{r}}} \exists w_{\alpha_{i}, \beta_{j}, \ldots, \lambda_{m}, \mu_{r}}^{k_{\alpha_{r}}}\right. \\
& \left(\left(w_{\alpha_{i}, \beta_{j}, \ldots, \lambda_{m}, \mu_{r}}^{k_{k_{i}}}=w_{\alpha_{i}, \beta_{j}, \ldots, \eta_{l}, \lambda_{m}}^{k_{a}}\right) \Rightarrow\right. \\
& \left.\Rightarrow\left(w_{\alpha_{i}, \beta_{j}, \ldots, \eta, \lambda_{m}}^{k_{a}}:=w_{\alpha_{i}, \beta_{j}, \ldots, \eta_{l}, \lambda_{m}}^{k_{a}} \cup \varnothing\right)\right) \vee \\
& \vee\left(\left(w_{\alpha_{i}, \beta_{j}, \ldots, \lambda_{m}, \mu_{r}}^{k_{a}} \cap w_{\alpha_{i}, \beta_{j}, \ldots, \lambda_{l}, \lambda_{m}}^{k_{a}} \neq \varnothing\right) \Rightarrow\right. \\
& \Rightarrow\left(w_{\alpha_{i}, \beta_{j}, \ldots, \eta, \lambda_{m}}^{k_{a}}:=w_{\alpha_{i}, \beta_{j}, \ldots, \eta_{l}, \lambda_{m}}^{k_{a}} \cup\right. \\
& \left.\left.\cup\left(w_{\alpha_{i}, \beta_{j}, \ldots, \lambda_{m}, \mu_{r}}^{k_{k}} \backslash w_{\alpha_{i}, \beta_{j}, \ldots, \eta_{l}, \lambda_{m}}^{k_{a}}\right)\right)\right) \vee \\
& \vee\left(\left(w_{\alpha_{i}, \beta_{j}, \ldots, \lambda_{m}, \mu_{r}}^{k_{a}} \cap w_{\alpha_{i}, \beta_{j}, \ldots, n_{l}, \lambda_{m}}^{k_{a}}=\varnothing\right) \Rightarrow\right. \\
& \Rightarrow\left(w_{\alpha_{i}, \beta_{j}, \ldots, \eta_{l}, \lambda_{m}}^{k_{k}}:=w_{\alpha_{i}, \beta_{j}, \ldots, \eta_{l}, \lambda_{m}}^{k_{a}} \cup\right. \\
& \left.\left.\left.\cup w_{\alpha_{i}, \beta_{j}, \ldots, \lambda_{m}, \mu_{r}}^{k_{a}}\right)\right)\right) \Rightarrow\left(T D_{\alpha_{i}, \beta_{j}, \ldots, \eta_{l}, \lambda_{m}}^{\prime} \rightarrow\right. \\
& \left.\left.\rightarrow T D_{\alpha_{i}, \beta_{j}, \ldots, \eta_{l}, \lambda_{m}}\right)^{\mathrm{W}}\right)
\end{aligned}
$$

8. On the definition of a fully formed holistic concept on the basis the additivity of the set of cost characteristics:

$$
\begin{aligned}
& \exists T D_{\alpha_{i}, \beta_{j}, \ldots, \eta_{l}, \lambda_{m}}^{\prime} \exists \mathrm{YTD}_{\mu} D_{\alpha_{i}, \beta_{j}, \ldots, \lambda_{m}, \mu} \\
& \left(\left(\mathrm{Y} \mathrm{H}_{\alpha_{i}, \beta_{j}, \ldots, \lambda_{m}, \mu}=H_{\alpha_{i}, \beta_{j}, \ldots, \eta_{l}, \lambda_{m}} \mid \forall z \in[1, k],\right.\right. \\
& \left(\sum_{\mu=1}^{n}\left\{h_{\alpha_{i}, \beta_{j}, \ldots, \lambda_{m}, \mu}^{z}\right\}=\left\{h_{\alpha_{i}, \beta_{j}, \ldots, \eta_{l}, \lambda_{m}}^{z}\right\}\right) \Rightarrow \\
& \left.\Rightarrow\left(T D_{\alpha_{i}, \beta_{j}, \ldots, \eta_{l}, \lambda_{m}}^{\prime} \rightarrow T D_{\alpha_{i}, \beta_{j}, \ldots, \eta_{l}, \lambda_{m}}\right)^{H}\right)
\end{aligned}
$$

IV. ALGORITHMIC PROCEDURES FOR CONSTRUCTING ONTOLOGICAL REPRESENTATIONS OF TECHNOLOGIES

The process of constructing a formalized description of technologies is the process of implementing formulated and proved theoretical propositions, which leads to the possibility of the formation of the DST by means of a staged combined design of the UDC, starting with the root representations of the technologies [21].

The implementation of main stages of UDC construction goes in accordance with the execution of the following algorithmic procedures (dotted line in "Fig. 2"):

1. The algorithm for the automated construction of decomposition of a preliminary formed concept (stage of downward design);

2. The algorithm for the automatic determination of relations between fully formed private concepts (stage of intra-level design);

3. The algorithm for the automatic determination of the complete formation of a holistic concept (stage of upward design).
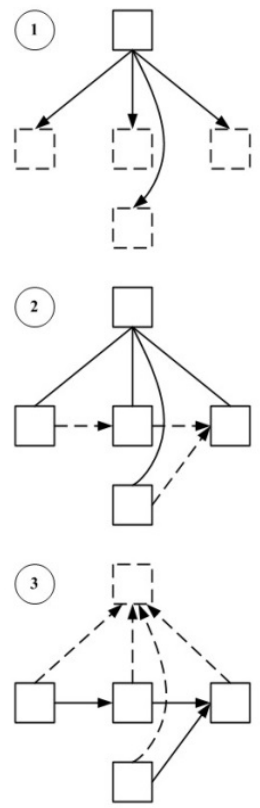

Fig. 2. Staged algorithmic procedures for constructing UDC

The stage of downward design is associated with the transition from a preliminary formed holistic concept to its representation as a set of private concepts of various initial degree of content formation. The methodological basis of this stage is the position of the axiomatic property 3.

The stage of intra-level design is responsible for establishing the relation of immediate precedence 
among the received fully formed private concepts. Methodologically this stage is provided by the positions of theoretical propositions 1-3.

Proposition 1. If the set $Y_{\alpha_{i}, \beta_{j}, \ldots, \lambda_{m}, \mu_{r}}$ of the fully formed concept $T D_{\alpha_{i}, \beta_{j}, \ldots, \lambda_{m}, \mu_{r}}$ is equal to the set $X_{\alpha_{i}, \beta_{j}, \ldots, \lambda_{m}, \mu_{s}}$ of the fully formed concept $T D_{\alpha_{i}, \beta_{j}, \ldots, \lambda_{m}, \mu_{s}}$, then the fully formed concept $T D_{\alpha_{i}, \beta_{j}, \ldots, \lambda_{m}, \mu_{r}}$ is the only immediately preceding concept for the fully formed concept $T D_{\alpha_{i}, \beta_{j}, \ldots, \lambda_{m}, \mu_{s}}:$ :

$$
\begin{aligned}
& \forall T D_{\alpha_{i}, \beta_{j}, \ldots, \lambda_{m}, \mu_{r}} \forall T D_{\alpha_{i}, \beta_{j}, \ldots, \lambda_{m}, \mu_{s}} \\
& \left(\left(Y_{\alpha_{i}, \beta_{j}, \ldots, \lambda_{m}, \mu_{r}}=X_{\alpha_{i}, \beta_{j}, \ldots, \lambda_{m}, \mu_{s}}\right) \Rightarrow\right. \\
& \Rightarrow\left(\exists ! T D_{\alpha_{i}, \beta_{j}, \ldots, \lambda_{m}, \mu_{r}},\right. \\
& \left.\left.\left(T D_{\alpha_{i}, \beta_{j}, \ldots, \lambda_{m}, \mu_{r}} P T D_{\alpha_{i}, \beta_{j}, \ldots, \lambda_{m}, \mu_{s}}\right)\right)\right)
\end{aligned}
$$

Proof. By definition 3 it follows that the fully formed concept $T D_{\alpha_{i}, \beta_{j}, \ldots, \lambda_{m}, \mu_{r}}$ is in the relation $P$ with the fully formed concept $T D_{\alpha_{i}, \beta_{j}, \ldots, \lambda_{m}, \mu_{s}}$.

Uniqueness is proved by the method of the opposite. Suppose that there is some fully formed concept $T D_{\alpha_{i}, \beta_{j}, \ldots, \lambda_{m}, \mu_{q}}$, which is also in the relation $P$ with the fully formed concept $T D_{\alpha_{i}, \beta_{j}, \ldots, \lambda_{m}, \mu_{s}}$. However, as a result of comparison of definition 3 for the fully formed concept $T D_{\alpha_{i}, \beta_{j}, \ldots, \lambda_{m}, \mu_{q}}$ with the first part of the proof of this proposition there is a contradiction with the position of the axiomatic property 1 . Therefore, the assumption made is incorrect and the fully formed concept $T D_{\alpha_{i}, \beta_{j}, \ldots, \lambda_{m}, \mu_{r}}$ is the only immediately preceding concept for the fully formed concept $T D_{\alpha_{i}, \beta_{j}, \ldots, \lambda_{m}, \mu_{s}}$.

Proposition 2. If the set $Y_{\alpha_{i}, \beta_{j}, \ldots, \lambda_{m}, \mu_{r}}$ of the fully formed concept $T D_{\alpha_{i}, \beta_{j}, \ldots, \lambda_{m}, \mu_{r}}$ is an own subset of the set $X_{\alpha_{i}, \beta_{j}, \ldots, \lambda_{m}, \mu_{s}}$ of the fully formed concept $T D_{\alpha_{i}, \beta_{j}, \ldots, \lambda_{m}, \mu_{s}}$ and there is no set of such fully formed concepts $\mathrm{Y}^{n} T D_{\alpha_{i}, \beta_{j}, \ldots, \lambda_{m}, \mu_{v_{k}}}$, that the totality of sets $\mathrm{Y}_{k=1}^{n} Y_{\alpha_{i}, \beta_{j}, \ldots, \lambda_{m}, \mu_{v_{k}}}$ is a subset of the complement $\mid X_{\alpha_{i}, \beta_{j}, \ldots, \lambda_{m}, \mu_{s}}^{*}$ of the set $X_{\alpha_{i}, \beta_{j}, \ldots, \lambda_{m}, \mu_{s}}$ of the fully formed concept $T D_{\alpha_{i}, \beta_{j}, \ldots, \lambda_{m}, \mu_{s}}$, then the fully formed concept $T D_{\alpha_{i}, \beta_{j}, \ldots, \lambda_{m}, \mu_{r}}$ is the only immediately preceding concept for the fully formed concept $T D_{\alpha_{i}, \beta_{j}, \ldots, \lambda_{m}, \mu_{s}}:$

$$
\begin{aligned}
& \forall T D_{\alpha_{i}, \beta_{j}, \ldots, \lambda_{m}, \mu_{r}} \forall T D_{\alpha_{i}, \beta_{j}, \ldots, \lambda_{m}, \mu_{s}} \\
& \left(\left(\left(Y_{\alpha_{i}, \beta_{j}, \ldots, \lambda_{m}, \mu_{r}} \subset X_{\alpha_{i}, \beta_{j}, \ldots, \lambda_{m}, \mu_{s}}\right) \wedge\right.\right.
\end{aligned}
$$$$
\wedge\left(\bar{\exists} \mathrm{Y}_{k=1}^{n} T D_{\alpha_{i}, \beta_{j}, \ldots, \lambda_{m}, \mu_{v_{k}}},\right.
$$

$$
\begin{aligned}
& \left(\mathrm { Y } _ { k = 1 } ^ { n } Y _ { \alpha _ { i } , \beta _ { j } , \ldots , \lambda _ { m } , \mu _ { v _ { k } } } \subseteq \left(X_{\alpha_{i}, \beta_{j}, \ldots, \lambda_{m}, \mu_{s}} \backslash\right.\right. \\
& \left.\left.\left.\left.\backslash X_{\alpha_{i}, \beta_{j}, \ldots, \lambda_{m}, \mu_{s}}^{*}\right)\right)\right)\right) \Rightarrow\left(\exists ! T D_{\alpha_{i}, \beta_{j}, \ldots, \lambda_{m}, \mu_{r}}\right. \\
& \left.\left.\left(T D_{\alpha_{i}, \beta_{j}, \ldots, \lambda_{m}, \mu_{r}} P T D_{\alpha_{i}, \beta_{j}, \ldots, \lambda_{m}, \mu_{s}}\right)\right)\right)
\end{aligned}
$$

Proof. By definition 3 it follows that the fully formed concept $T D_{\alpha_{i}, \beta_{j}, \ldots, \lambda_{m}, \mu_{r}}$ is in the relation $P$ with the fully formed concept $T D_{\alpha_{i}, \beta_{j}, \ldots, \lambda_{m}, \mu_{s}}$.

The proof of uniqueness is constructed by correlating the position of the axiomatic property 4 and the conditional part of this proposition for the fully formed concepts $T D_{\alpha_{i}, \beta_{j}, \ldots, \lambda_{m}, \mu_{r}}$ and $T D_{\alpha_{i}, \beta_{j}, \ldots, \lambda_{m}, \mu_{s}}$. As a result of the analysis, it turns out that the subset of the set $X_{\alpha_{i}, \beta_{j}, \ldots, \lambda_{m}, \mu_{s}}$ is exclusively the set $Y_{\alpha_{i}, \beta_{j}, \ldots, \lambda_{m}, \mu_{r}}$ , which by the axiomatic property 1 is unique. Thus, the fully formed concept $T D_{\alpha_{i}, \beta_{j}, \ldots, \lambda_{m}, \mu_{r}}$ is the only immediately preceding concept for the fully formed concept $T D_{\alpha_{i}, \beta_{j}, \ldots, \lambda_{m}, \mu_{s}}$.

The situation when such set of fully formed $\mathrm{Y}_{k=1}^{n} T D_{\alpha_{i}, \beta_{j}, \ldots, \lambda_{m}, \mu_{v_{k}}}$ exists, the absence of which is stated in the position of the proposition 2 , is considered in the proposition 3 . In that case, the investigative part of the position of the proposition 3 contains a conclusion about the multiplicity of immediate precedence to the fully formed concept $T D_{\alpha_{i}, \beta_{i}, \ldots, \lambda_{m}, \mu_{s}}$. This proposition is proved by a similar scheme of the given proof of the proposition 2 .

The final stage of UDC construction is the stage of upward design, which is the establishing relation of "partwhole" between each of the fully formed private concepts and the already fully formed holistic concept. The implementation of the corresponding algorithm is methodologically determined by the position of the proposition 4 .

Proposition 4. If all the concepts $\mathrm{YTD}_{\mu} D_{\alpha_{i}, \beta_{j}, \ldots, \lambda_{m}, \mu}$ are fully formed, and for any concept $T D_{\alpha_{i}, \beta_{j}, \ldots, \lambda_{m}, \mu_{r}}$ there is no such concept $T D_{\alpha_{i}, \beta_{j}, \ldots, \lambda_{m}, \mu_{q}}$, that the set $Y_{\alpha_{i}, \beta_{j}, \ldots, \lambda_{m}, \mu_{q}}$ of the concept $T D_{\alpha_{i}, \beta_{j}, \ldots, \lambda_{m}, \mu_{q}}$ is not a subset of the set $X_{\alpha_{i}, \beta_{j}, \ldots, \lambda_{m}, \mu_{r}}$ of the concept $T D_{\alpha_{i}, \beta_{i}, \ldots, \lambda_{m}, \mu_{r}}$, and there is also no such concept $T D_{\alpha_{i}, \beta_{j}, \ldots, \lambda_{m}, \mu_{s}}^{\alpha_{i}, \beta_{i}, \ldots, \lambda_{m}, \mu_{r}}$, that the set $Y_{\alpha_{i}, \beta_{j}, \ldots, \lambda_{m}, \mu_{r}}$ of the concept $\mathfrak{D}_{\alpha_{i}, \beta_{j}, \ldots, \lambda_{m}, \mu_{r}}$ is not a subset of the set 
$X_{\alpha_{i}, \beta_{j}, \ldots, \lambda_{m}, \mu_{s}}$ of the concept $T D_{\alpha_{i}, \beta_{j}, \ldots, \lambda_{m}, \mu_{s}}$ , then each of the concepts $\mathrm{YTD}_{\mu} \mathrm{\alpha}_{\alpha_{i}, \beta_{j}, \ldots, \lambda_{m}, \mu}$ is a private concept for the fully formed holistic concept

$$
\begin{aligned}
& T D_{\alpha_{i}, \beta_{j}, \ldots, \eta_{l}, \lambda_{m}}: \\
& \exists T D_{\alpha_{i}, \beta_{j}, \ldots, \eta_{l}, \lambda_{m}} \exists \mathrm{YTD}_{\mu} T D_{\alpha_{i}, \beta_{j}, \ldots, \lambda_{m}, \mu} \\
& \left(\left(\forall T D_{\alpha_{i}, \beta_{j}, \ldots, \lambda_{m}, \mu_{r}}\right.\right. \\
& \quad \bar{\exists} T D_{\alpha_{i}, \beta_{j}, \ldots, \lambda_{m}, \mu_{q}} \bar{\exists} T D_{\alpha_{i}, \beta_{j}, \ldots, \lambda_{m}, \mu_{s}} \\
& \left(\left(\neg\left(Y_{\alpha_{i}, \beta_{j}, \ldots, \lambda_{m}, \mu_{q}} \subseteq X_{\alpha_{i}, \beta_{j}, \ldots, \lambda_{m}, \mu_{r}}\right)\right) \wedge\right. \\
& \left.\left.\wedge\left(\neg\left(Y_{\alpha_{i}, \beta_{j}, \ldots, \lambda_{m}, \mu_{r}} \subseteq X_{\alpha_{i}, \beta_{j}, \ldots, \lambda_{m}, \mu_{s}}\right)\right)\right)\right) \Rightarrow \\
& \left.\Rightarrow\left(\forall \mathrm{YT}_{\alpha_{\alpha_{i}, \beta_{j}, \ldots, \lambda_{m}, \mu}} F T D_{\alpha_{i}, \beta_{j}, \ldots, \eta_{l}, \lambda_{m}}\right)\right)
\end{aligned}
$$

Proof. There is a sufficient number of possible cases in the proof of this proposition, which is associated to the total quantity and mutual arrangement of the fully formed concepts $\mathrm{Y}_{k=1}^{n} T D_{\alpha_{i}, \beta_{j}, \ldots, \lambda_{m}, \mu_{v_{k}}}$. In this regard, the proof will be conducted on the example of "Fig. 1", when $n=4$.

The determination of the complete formation of the holistic concept $T D_{\alpha_{i}, \beta_{j}, \ldots, \eta_{l}, \lambda_{m}}$ is based on the conditional part of this proposition and the positions of the axiomatic properties 6-8, and there are some special features. They are associated with the application of the position of the axiomatic property 6 , according to which it turns out that: a) the set $X_{\alpha_{i}, \beta_{j}, \ldots, \eta_{l}, \lambda_{m}}$ of the holistic concept $T D_{\alpha_{i}, \beta_{j}, \ldots, \eta_{l}, \lambda_{m}}$ is supplemented by the set $X_{\alpha_{i}, \beta_{j}, \ldots, \lambda_{m}, \mu_{v_{I}}}$ of the fully formed private concept $T D_{\alpha_{i}, \beta_{j}, \ldots, \lambda_{m}, \mu_{v j}}$, which by definition 4 is initial;

b) since the fully formed private concept $T D_{\alpha_{i}, \beta_{j}, \ldots, \lambda_{m}, \mu_{v_{j}}}$ is the only immediately preceding concept for the fully formed private concept $T D_{\alpha_{i}, \beta_{j}, \ldots, \lambda_{m}, \mu_{v_{2}}}$, at that $Y_{\alpha_{i}, \beta_{j}, \ldots, \lambda_{m}, \mu_{v_{1}}}=X_{\alpha_{i}, \beta_{j}, \ldots, \lambda_{m}, \mu_{v_{2}}}$ , the set $X_{\alpha_{i}, \beta_{j}, \ldots, \eta_{l}, \lambda_{m}}$ from the set $X_{\alpha_{i}, \beta_{j}, \ldots, \lambda_{m}, \mu_{v 2}}$ is not replenished by anything; c) the set $X_{\alpha_{i}, \beta_{j}, \ldots, \eta_{l}, \lambda_{m}}$ of the holistic concept $T D_{\alpha_{i}, \beta_{j}, \ldots, \eta_{l}, \lambda_{m}}$ is supplemented by the set $X_{\alpha_{i}, \beta_{j}, \ldots, \lambda_{m}, \mu_{v_{3}}}$ of the fully formed private concept $T D_{\alpha_{i}, \beta_{j}, \ldots, \lambda_{m}, \mu_{v_{3}}}$, which by definition 6 is complementary; d) since the union of the sets $Y_{\alpha_{i}, \beta_{j}, \ldots, \lambda_{m}, \mu_{v_{2}}}$ and $Y_{\alpha_{i}, \beta_{j}, \ldots, \lambda_{m}, \mu_{v_{3}}}$ is an own subset of the set $X_{\alpha_{i}, \beta_{j}, \ldots, \lambda_{m}, \mu_{v_{4}}}$, the set $X_{\alpha_{i}, \beta_{j}, \ldots, \eta_{l}, \lambda_{m}}$ of the holistic concept $T D_{\alpha_{i}, \beta_{j}, \ldots, \eta_{l}, \lambda_{m}}$ is supplemented by the complement $\mid X_{\alpha_{i}, \beta_{j}, \ldots, \lambda_{m}, \mu_{v_{4}}}^{*}$ of the set $X_{\alpha_{i}, \beta_{j}, \ldots, \lambda_{m}, \mu_{v_{4}}}$ of the fully formed private concept $T D_{\alpha_{i}, \beta_{j}, \ldots, \lambda_{m}, \mu_{v_{4}}}$.

Thus, by definition 7 each of the fully formed concepts 4 $\mathrm{Y}_{k=1} T D_{\alpha_{i}, \beta_{j}, \ldots, \lambda_{m}, \mu_{v_{k}}}$ is in the relation of "part-whole" with the already fully formed concept $T D_{\alpha_{i}, \beta_{j}, \ldots, \eta_{l}, \lambda_{m}}$ , while the requirements of the axiomatic property 2 are also met for the fully formed holistic concept $T D_{\alpha_{i}, \beta_{j}, \ldots, \eta_{l}, \lambda_{m}}$.

For other quantities and mutual arrangements of the fully formed concepts $\mathrm{YTD}_{\mu} \mathrm{\alpha}_{\alpha_{i}, \beta_{j}, \ldots, \lambda_{m}, \mu}$, the proof of this proposition will be constructed on a similar scheme.

\section{CONCLUSION}

By the results of consideration of the proposed models' features and the constructed algorithms it can be concluded that as a result the method of formalized description of technologies was developed, differing from the existing methods by presence of the possibility of the formation of the DST by means of the staged combined design of the UDC, with the purpose of structuring of knowledge representation about technologies with various degree of detail.

The distinctive feature of the proposed model of the concept of technological action is the concentration of all the semantics of technological actions, located in the nodes of the DST, exclusively within the sets that are part of the structure of these conceptual formations.

The specificity of the constructed model of the formalized description of technologies is in organizing the process of the formation of the DST, with the purpose of reception of analytical tools for ontological representations of technologies by determining and establishing all the entered relations, based on the constructive features of the embedded model of the concept of technological action. The axiomatic component of this model determines the basic laws of UDC construction, from which the DST are already formed.

The distinctive features of the developed algorithms include: constructing ontological hierarchies of concepts basing on predetermined signs of decomposition, automatic establishment of relations between concepts of the same level of decomposition and automatic receiving full information about the concepts located in the root nodes of the DST, based on the implemented principle of level-by-level aggregation of knowledge. 


\section{REFERENCES}

[1] R. Koller and N. Kastrup, Prinziplösungen zur konstruktion technischer produkte. Berlin: Springer, 1994.

[2] R. J. Van Wyk, ,Technology: a fundamental structure, “Knowledge, Technology, and Policy, vol. 15 (3), pp. 14-35, 2002. V. V. Sadovsky, M. V. Samoilov, and N. P. Kokhno, Production technologies. Minsk: BSEU, 2008. (in Russian)

[3] D. A. Makhotin, "Technological knowledge in modern education," Bulletin of Moscow state regional University, Pedagogics, No. 2, pp. 116-121, 2010. (in Russian)

[4] D. A. Andreev, "Methods of formalized description of technologies: an attempt to overview," Proceedings of Pskov Polytechnic Institute, Mechanical engineering. Electrical engineering, No. 15.3, pp. 291-297, 2011. (in Russian)

[5] S. Staab and R. Studer, Handbook on ontologies. Berlin: Springer, 2004.

[6] A. V. Palagin and N. G. Petrenko, "System-ontological analysis of the subject area," Control systems and machines, No. 4, pp. 3-14, 2009. (in Russian)

[7] I. V. Antonov and M. V. Voronov, "Methods of data analysis in the automation tasks of domain ontology," Distance and virtual training, No. 8, pp. 19-35, 2011. (in Russian)

[8] G. B. Evgenev, "The ontological methodology for creating intelligent systems in mechanical engineering," Proceedings of higher educational institutions, Mechanical engineering, vol. 6 (651), pp. 39-47, 2014. (in Russian)

[9] V. B. Artemenko and A. A. Ivlev, "The ontology of military technologies based on conceptual maps," Investigated in Russia, vol. 14, pp. 285-294, 2011. (in Russian)

[10] R. A. Calvo and J. Villalon, „Concept maps as cognitive visualizations of writing assignments, “ Educational technology and society, vol. 14 (3), pp. 16-27, 2011.

[11] R. Carnap, „Empiricism, Semantics, and Ontology,“ Revue Internationale de Philosophie, vol. 4, pp. 20-40, 1950.
[12] V. F. Turchin, ,The cybernetic ontology of actions, “ Kybernetes, vol. 22 (2), pp. 10-30, 1993

[13] D. A. Andreev, "Model of conceptual elements of activity in the formalized descriptions of production technologies," in proceedings of the XXXV International scientific and practical conference on prospects of development of information technologies, Novosibirsk, 2017, pp. 6-13. (in Russian)

[14] D. A. Andreev, "Decomposition representation of knowledge as the basis of ontological engineering of technological processes," in proceedings of the second international scientific and technical conference on computer science and technology, Belgorod, 2011, pp. 140-144. (in Russian)

[15] T. R. Gruber, ,A translation approach to portable ontology specifications," Knowledge acquisition, vol. 5 (2), pp. 199-220, 1993.

[16] S. V. Maltseva, "Application of ontological models for solving identification problems and domain monitoring," Business Informatics, No. 3, pp. 18-24, 2008. (in Russian)

[17] B. V. Dobrov and N. V. Lukashevich, "Transitive non-taxonomic relations in ontological modeling," in proceedings of the symposium on ontological modeling, Zvenigorod, 2008, pp. 229-259. (in Russian)

[18] D. A. Andreev and M. V. Voronov, "Model of unified constructions of technology description in their ontological representations," in proceedings of the XXVI international scientific conference on mathematical methods in engineering and technologies, Nizhny Novgorod, 2013, vol. 8, pp. 138-140. (in Russian)

[19] D. A. Andreev, "Axiomatics of the model of formalized description of arbitrary technology," in proceedings of the XXVIII international scientific conference on mathematical methods in engineering and technologies, Saratov, 2015, vol. 4, pp. 77-80. (in Russian)

[20] D. A. Andreev and M. V. Voronov, "Procedural mechanism for constructing ontological representations of the decomposition structures of technologies," in proceedings of the XV all-Russian joint conference on the Internet and Modern Society, Saint-Petersburg, 2012, pp. 37-40. (in Russian) 